Mauricio Gutiérrez Ortega 2

\section{Estrategias campesinas de reproducción económica y paludismo en la microregión pliegues fallados de los Altos de Chiapas, México: estudio de caso 1}

\author{
Peasant strategies for economic reproduction \\ and malaria epidemiology in the ravines \\ microregion of the Chiapas mountains, \\ Mexico: a case study 1
}

1 Investigación realizada con apoyo de CONACYT, proyecto “Agroecología, Aspectos Productivos y Malaria en el Municipio de Chenalhó, Chiapas" no 1522-M9207 2 Departamento de Microbiología y Parasitología, Facultad de Medicina, Universidad Nacional Autónoma de México. Calle 28 de agosto ㄲo 11 , San Cristóbal de Las Casas, Chiapas, 29200, México.
Abstract The goal of this investigation was to establish an initial correlation between farming activities and malaria and to definerisk factors and prevalence of the latter through an analysis of the integration of farm production strategies by members of an indigenous peasant community in the Chiapas mountains in Mexico. Information was obtained on places of work, land use, coffee, corn, and bean farming, and number of family members involved in farming activities, wage labor, and handi crafts production. Migration of farm workers to warmer climates was also analyzed. The study compared families with and without cases of malaria from 1987 to 1993 in the town of Yibeljoj, Chenal hó county. The most outstanding characteristics of this analysis were the following: strategies invol ving greater risk and prevalence of malaria were those which combined corn farming and wage labor; on the other hand, strategies in which handicraft production was the activity of primary or secundary importance were associated with few or no cases of the disease.

Key words Malaria; Epidemiology; Risk; Economic Estrategies; Peasant Soci eties

Resumen El objetivo de esta investigación fue establecer relaciones iniciales entre actividad agrícola y paludismo, definíendoseriesgos y prevalencia a través del análisis dela integración de estrategi as de reproducción de los pobladores de una comuni dad indígena de Los Altos de Chiapas. Se obtuvo información sobre si tios de trabajo, uso del suelo, volúmenes de producción de café, maíz y frijol y número de unidades familiares involucradas en esta producción, el trabajo asal ariado y en las artesanías. Se anal izó la circulación productiva a los "trabajaderos" detierra caliente. Se compararon unidades familiares con casos y sin casos de paludismo, entre 1987 y 1993, en Ybeljoj, Munici pio de Chenal hó, Chiapas. Entre los resultados más sobresal ientes encontramos que las estrategias que ofreci eron mayores riesgos y prevalencia de paludismo fueron aquellas donde la actividad del cultivo del maíz y el trabajo asalariado estaban presentes; contrariamente, aquellas estrategias en donde la artesanía era la actividad principal o de segunda importancia estaban asociadas con la ausencia del paludismo o prevalencia mínima de la enfermedad.

Palabras clave Malaria; Epidemiología; Riesgo; Estrategias Económicas; Sociedades Campesianas 


\section{Objetivos de la investigación}

El estudio pretendió caracterizar, de manera general, condiciones socioeconómicas prevalecientes en el ámbito rural y al gunas condiciones de trabajo en los espacios productivos llamados "trabajaderos". Con ello estaremos dando cuenta, en forma inicial, cómo la situación de marginación extrema (en comunidades con pobre desarrollo socioeconómico) conforma cuadros epidemiológicos de alta endemicidad, entre otros el del paludismo.

En forma particular, tratamos de demostrar cómo, al interior de cada comunidad, las diferencias en condiciones económicas y por ende de condiciones de vida y trabajo de los campesinos acarrean diferencias en la endemia palúdica posibilitando una herramienta de tipo metodológico, a fin de ubicar el grado de causalidad de la falta de desarrollo de estas comunidades $y$, en particular, en los diferentes estratos sociales, en la instalación y efecto de endemias rurales como el paludismo.

El resurgimiento del paludismo en diferentes países y regiones

La Organización Panamericana de la Salud (OPS-OMS, 1990) indica que a nivel continental, la ubicación geográfica de los países tiene estrecha relación con condiciones ecológicas comunes, la historia, la idiosincrasia, la cultura y las costumbres de la población, favoreciendo la interacción de factores bioambientales, demográficos y socio-económicos que influyen en la transmisión de la malaria. Por otro lado, Loyola (1985), al analizar la naturaleza de los problemas que explican los altos incrementos de casos de paludismo en muchos países del área de las Américas (entre ellos M éxico), señala que la magnitud y el tipo de problemas que han originado esos incrementos difieren según países y regiones, pero que tienen rasgos comunes que resume así: “....problemas técnicos, que incluyen la resistencia fisiológica y etológica... de los anofelinos ante los insecticidas... Otros son los problemas de desarrollo socioeconómico y cultural como vivienda precaria..., los movimientos de población..., las prácticas relacionadas con la toma de antipalúdicos, y la renuencia al rociado de insecticidas residuales en las viviendas".

Nuestra postura, en términos generales, se identifica con tales análisis y consideramos que como realidad compleja, no solo bioecológica sino también de índoles socioeconómica, el paludismo tiene una multiplicidad de deter- minaciones y condicionantes que se hace necesario esclarecer en estudios específicos de cada región y aún de microregiones.

Hasta aquí nuestra posición está referida a aquellos factores o variables de determinación que consideramos de mayor relevancia. Esto no implica que también estemos de acuerdo con aspectos sostenidos por quienes incluyen los factores técnicos como parte de la problemática. Entre los que le dan mayor importancia a este tipo de factores, podemos citar a Fernández de Castro (1988) y a González-Casanova (1994), a quienes ubicaríamos dentro de la orientación un tanto reduccionista de ver la problemática. El primero de ellos, al omitir de los contenidos de su valioso documento " $\mathrm{Pa}$ norama Histórico y Epidemiológico del Paludismo en México" toda la causalidad socioeconómica, demográfica, cultural y sociopolítica de la problemática. El segundo, al privilegiar muy sectorialmente las variables técnicas, y de estas una sola (políticas del rociado del DDT), como orígen y causa del resurgimiento del paludismo.

\section{Situación geográfica de la microregión} "Pliegues Fallados"

De acuerdo a Mera (1989), el sistema terrestre denominado “Pliegues Fallados Huixtán-Larraínzar" que, en este estudio, el territorio que incluye fue considerado como microregión, es parte integrante de la subregión San Cristóbal, la que, a su vez, a diferencia del resto de la región Altos de Chiapas, por su relativa homogeneidad ecológica, uso del suelo y características culturales y étnicas, se integra económicamente a la ciudad del mismo nombre. La mayor parte de la microregión se ubica entre los 160 17' y 17o Latitud Norte y los 920 07' y 920 49' Longitud Oeste (Figura 1).

Sólo algunas zonas no muy extensas de estas regiones que nos ocupan reúnen los factores climáticos de temperatura, Iluvias y humedad para permitir la existencia y desarrollo de suficientes densidades de mosquitos susceptibles a la infección por plasmodios (agentes etiológicos del paludismo) y el cumplimiento del ciclo de vida de estos agentes causales de la enfermedad (Forattini,1962).

En relación a las condiciones climáticas y datos de incidencia estacional del paludismo en la microregión (SSA, 1993), los tipos geográfico-epidemiológicos de esta enfermedad serían: Paludismo Subtropical y Paludismo Paraequatorial (Forattini, 1962). El primer tipo presente en las zonas de vivienda entre los 
1200 y $1600 \mathrm{~m}$ y, el segundo, en los “trabajaderos" de maíz-frijol en altitudes de 400 a 900 m.

\section{Estrategias campesinas de reproducción económica}

Respecto a nuestro objeto de estudio - una economía campesina de un municipio indígena de la subregión San Cristóbal, en Los Altos de Chiapas -, las estrategias se caracterizarían por relaciones mercantiles que han penetrado desde hace tiempo a tales formas campesinas de producir bienes materiales, los que, en grado importante, serían mercancías destinadas a conseguir ingresos y, desde luego, bienes de autoconsumo, con mayor o menor importancia para la reproducción de la fuerza de trabajo.

Otra actividad de suma importancia para la generación de ingresos y componentes para la reproducción de las unidades familiares sería, precisamente, el asalaramiento o venta de fuerza de trabajo. Así, todas las actividades desarrolladas para garantizar esa reproducción de fuerza de trabajo, sensu estrictu - reproducción biológica de los productores y su familia -, en general también para garantizar la vida social de los integrantes de unidades familiares campesinas, conformarían modos y formas de combinarse en el tiempo y en espacios - con determinados medios de producción - para constituir estrategias de reproducción económicas.

En resumen, las actividades productivas agrícolas (fueran para generar alimentos de autoconsumo o productos para la venta), artesanales, la venta de fuerza de trabajo, integrarían, combinadas en tiempos, espacios y grados, estrategias económicas de reproducción de las unidades familiares.

\section{Identificación de estrategias en la comunidad de Yibeljoj}

Con el propósito de analizar empíricamente estrategias de reproducción y su relación con el paludismo, realizamos un estudio en la comunidad deYibeljoj, del municipio de ChenaIhó, la cual consideramos representativa desde el punto de vista productivo y socioeconómico del resto de comunidades del municipio. En esta comunidad se estudiaron 94 Unidades Familiares (productivas), 47 de estas con algún caso de paludismo registrado entre 1987 y 1993 y 47 sin ningún caso registrado en ese mismo período.

El número identificado de Estrategias de Reproducción Económica (ERE) fue de dieci-
Figura 1

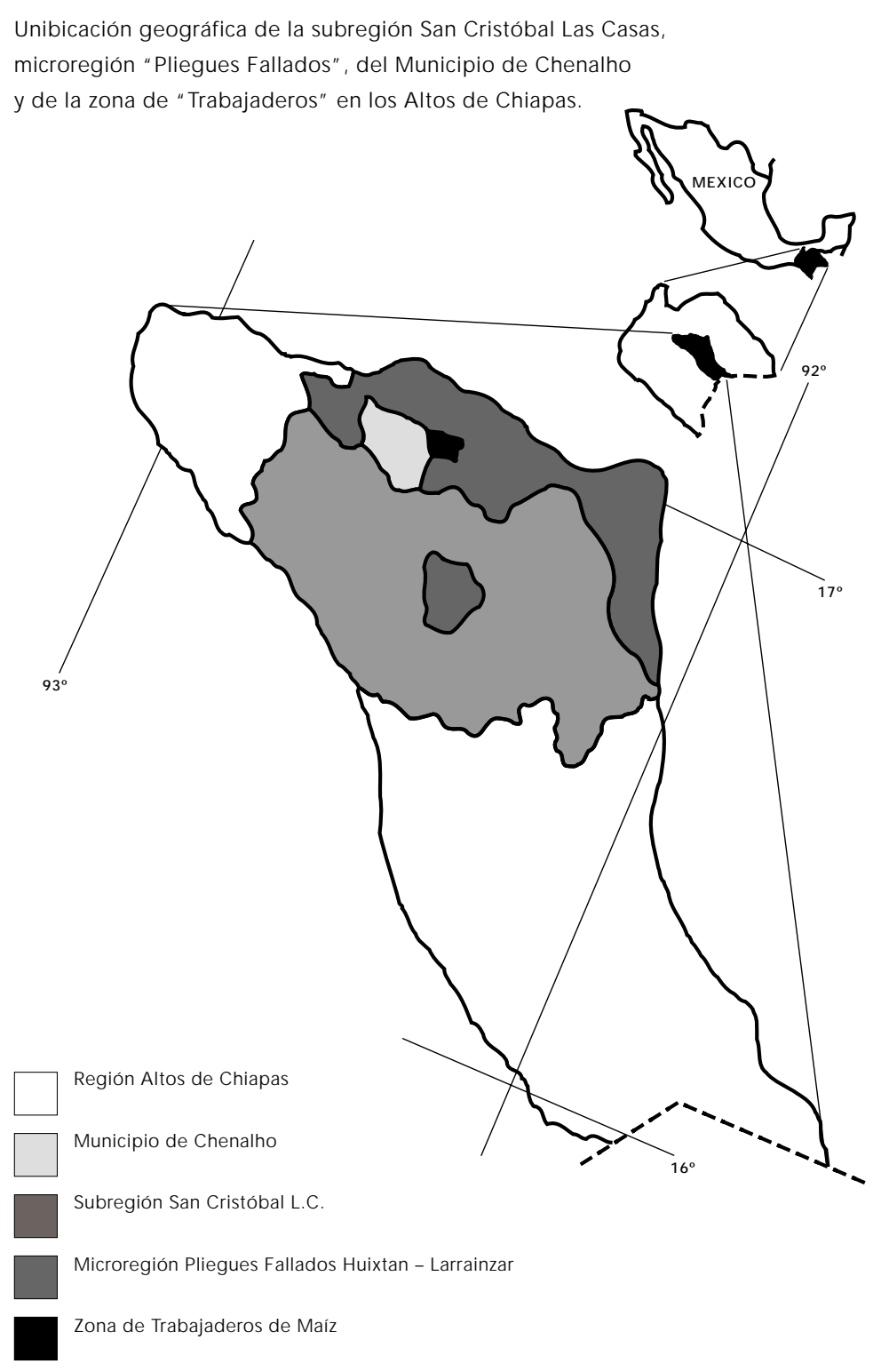

Fuente: Mapa trabajado por el autor en base a Mera, 1989.

séis, aunque para fines de la caracterización y de la relación con el paludismo nos centraremos en las principales. El criterio para ser consideradas principales fue el número de Unidades Familiares (UF) integrando a las ERE; para el caso, solo las ERE de cuatro y más unidades familiares fueron seleccionadas para los análisis citados.

Las estrategias de reproducción económica identificadas se enumeran en la Tabla 1.

De acuerdo a los componentes de actividad económica para cada una de las ERE, se cita, en primer término, la actividad que representó el mayor ingreso monetario $y$, en segundo lu- 
Tabla 1

Estrategias de reproducción económica en unidades familiares de Yibeljoj, Chenalhó, Chiapas.

\begin{tabular}{lrr}
\hline $\begin{array}{l}\text { Estrategias de } \\
\text { reproducción económica }\end{array}$ & $\mathrm{n}$ & $\%$ \\
\hline café-salario & 21 & 22,34 \\
café-maíz & 16 & 17,02 \\
artesanías-café & 15 & 15,97 \\
salario-café & 12 & 12,76 \\
café-frijol & 5 & 5,32 \\
salario-maíz & 5 & 5,32 \\
artesanías-maíz & 5 & 5,32 \\
salario solo & 3 & 3,19 \\
café solo & 2 & 2,13 \\
salario-artesanías & 2 & 2,13 \\
artesanías-salario & 2 & 2,13 \\
maíz-café & 2 & 2,13 \\
salario-frijol & 1 & 1,06 \\
artesanías-frijol & 1 & 1,06 \\
artesanías solo & 1 & 1,06 \\
maíz-salario & 1 & 1,06 \\
Totales & & \\
\hline UF (Unidades Familiar & 94 & 100,0 \\
\hline & &
\end{tabular}

UF (Unidades Familiares)

Fuente: Encuesta socioeconómica del autor. CIHMECH, 1994.

Tabla 2

Estrategias de reproducción económica y porcentaje de ingreso en Yibeljoj, Chenalhó, Chiapas, 1994.

\begin{tabular}{lrrrrrr}
\hline $\begin{array}{l}\text { Estrategias de } \\
\text { reproducción económica }\end{array}$ & \multicolumn{2}{c}{ UF } & \multicolumn{3}{c}{$\%$ del ingreso } \\
& $n$ & & UF & 1 & 2 \\
\hline café-salario & 21 & 22,34 & 87,68 & 60,08 & 27,60 \\
café-maíz & 16 & 17,02 & 91,29 & 74,88 & 16,41 \\
artesanías-café & 15 & 15,97 & 97,11 & 75,75 & 21,36 \\
salario-café & 12 & 12,76 & 86,26 & 62,80 & 23,48 \\
café-frijol & 5 & 5,32 & 91,40 & 76,52 & 14,88 \\
salario-maíz & 5 & 5,32 & 86,46 & 59,80 & 26,66 \\
artesanías-maíz & 5 & 5,32 & 92,32 & 83,30 & 9,02 \\
otras estrategias & 15 & 15,95 & 92,83 & 76,16 & 16,67 \\
& & & & & \\
Totales & 94 & 100,0 & 90,67 & 71,16 & 19,51 \\
\hline
\end{tabular}

UF (Unidades Familiares)

Fuente: Encuesta socioeconómica del autor. CIHMECH, 1994. gar, la actividad que le siguió en importancia como porcentaje del ingreso total anual dentro de las UF. Así, en la ERE café-salario, por ejemplo, el café representó el mayor porcentaje del ingreso en las UF de esa estrategia y el salario (trabajo asalariado), el porcentaje que le siguió en aporte del ingreso anual de las UF (Tabla 2).

Al analizarse los diferentes componentes o actividades económicas de las siete ERE mas importantes, el café resultó ser la principal actividad, apareciendo tres veces como el componente principal y dos veces como componente secundario. Le siguieron el trabajo asalariado y el maíz, los que aparecen tres veces en las siete estrategias principales, con la diferencia de que el trabajo asalariado se presenta en dos ocasiones como el componente principal. La artesanía solo aparece dos veces y lo hace como componente principal. Para valorar la importancia como proporción del ingreso anual de las UF en las diferentes ERE, ver la Tabla 2.

Respecto a la importancia por el número de UF integrando las ERE, podemos ver, en la Tabla 1, que las siete principales estrategias suman el $84,05 \%$ del total de UF de las 16 ERE.

Relación de las Estrategias de Reproducción Económica (ERE) con los riesgos y prevalencia del paludismo

Es conveniente mencionar que, en nuestras hipótesis al inicio del estudio, se consideraba que los espacios o sitios de trabajo agrícola-campesino representaban elementos importantes de riesgo de adquirir el paludismo. De estos espacios, sobresalían de manera especial los Ilamados "trabajaderos" de tierra caliente.

Otra consideración introductoria es que, metodológicamente, se procedió a la agrupación en dos conjuntos, los casos de paludismo registrados en la comunidad de Yibeljoj, Chenalhó entre 1987 y 1994 y los correspondientes no casos registrados, pareados por sexo, edad y barrio en igual número de unidades familiares que para los casos, dentro del total de pobladores de la comunidad mencionada. En la Tabla 3 , se presenta la distribución del número y porcentaje de Unidades Familiares en cada una de las Estrategias de Reproducción Económica, así como el número y porcentaje de UF con y sin casos distribuidos en cada una de las ERE.

Haciendo un análisis comparativo de ambos conjuntos, se encontraron elementos de juicio para sustentar los argumentos que se presentan en este apartado. Siendo el cultivo y la producción de maíz en los "trabajaderos" de "tierra caliente" y el trabajo asalariado las acti- 
Tabla 3

Estrategias de reproducción económica. número y porcentaje de unidades

familiares con casos y sin casos de paludismo, entre 1987 y 1993, en Yibeljoj, Chenalhó, Chiapas, 1994.

\begin{tabular}{|c|c|c|c|c|c|c|}
\hline \multirow[t]{2}{*}{$\begin{array}{l}\text { Estrategias de } \\
\text { reproducción económica }\end{array}$} & \multicolumn{2}{|c|}{ UF } & \multicolumn{2}{|c|}{$\begin{array}{l}\text { Con casos } \\
\text { de paludismo }\end{array}$} & \multicolumn{2}{|c|}{$\begin{array}{l}\text { Sin casos } \\
\text { de paludismo }\end{array}$} \\
\hline & $n$ & $\%$ & $\mathrm{n}$ & $\%$ & $\mathrm{n}$ & $\%$ \\
\hline café-salario & 21 & 22,34 & 10 & 47,6 & 11 & 52,4 \\
\hline café-maíz & 16 & 17,02 & 11 & 68,7 & 5 & 31,2 \\
\hline artesanías-café & 15 & 15,95 & 1 & 6,6 & 14 & 93,3 \\
\hline salario-café & 12 & 12,76 & 7 & 58,3 & 5 & 41,7 \\
\hline café-frijol & 5 & 5,32 & 1 & 20,0 & 4 & 80,0 \\
\hline salario-maíz & 5 & 5,32 & 5 & 100,0 & - & - \\
\hline artesanías-maíz & 5 & 5,32 & 5 & 100,0 & - & - \\
\hline otras estrategias & 15 & 15,95 & 7 & 60,0 & 8 & 53,3 \\
\hline Totales & 94 & 100,0 & 47 & & 47 & \\
\hline
\end{tabular}

UF (Unidades Familiares)

Fuente: Encuesta socioeconómica del autor. CIHMECH, 1994.

vidades económicas que resultaron asociadas al riesgo y prevalencia del paludismo entre las unidades campesinas de Yibeljoj, nos limitaremos a tratar de demostrar cómo algunas estrategias de reproducción económica donde se integran tales actividades - variables - representan y explican mayores riesgos y prevalencia del paludismo. Por otra parte, también se presentan argumentos de asociación causal aunque menos directos - sobre estrategias donde la artesanía y, en menor grado, el café acarrearían menos riesgos a la infección.

Estrategias de Reproducción Económica (ERE) y actividades agrícolas (en parcelas propias y como peones asalariados) en Ios "trabajaderos" de Unidades Familiares (UF) con casos y sin casos de paludismo

Anteriormente hemos mencionado la relación, todavía hipotética, entre las condiciones de transmisión de paludismo que ofrecen los “trabajaderos" y la presencia de campesinos con parcelas de maíz-frijol o como trabajadores vendiendo su fuerza de trabajo como peones. En las Tablas 4 y 5, se comparan niveles de estas presencias y actividades, observándose claras diferencias entre las UF y sus ERE de casos y las de no casos de paludismo. Por ejemplo, de las UF de los casos, ocho tienen parcelas de maízfrijol en esos "trabajaderos", contra sólo dos UF de los no casos; 18 UF de los casos tienen actividades en ambos ciclos de producción de maíz, contra sólo 10 y 6 UF de los no casos, en los ciclos 1 y 2 , respectivamente. Finalmente, respecto a la presencia como peones asalariados, ca- torce UF de casos contra una sola UF de no casos de paludismo la tienen en los "trabajaderos".

Observando los porcentajes de UF con casos y con actividades en los "trabajaderos" con venta de fuerza de trabajo, precisamente, los mayores porcentajes corresponden a las estrategias donde participa el trabajo asalariado como principal o secundaria fuente de ingreso: ERE café-salario, 43,7\%; ERE salario-café, $33,1 \%$ y ERE salario-maíz, 8,5\%. En cambio, en las UF de no casos, sólo una UF se asalaría en los "trabajaderos", lo que resulta muy significativo para fortalecer y confirmar la hipótesis de que, a mayor trabajo asalariado, sobre todo desarrollado en parcelas de maíz-frijol ubicadas en "Ios trabajaderos", mayores riesgos y prevalencia del paludismo.

Las Tablas 6 y 7 también reforzarían lo mencionado líneas arriba, pues quienes venden más fuerza de trabajo como número de UF y como jornales, independientemente de los sitios de venta de este, son precisamente las UF con casos de paludismo y en las estrategias donde el salario es componente principal o secundario: 32 UF venden su fuerza de trabajo y, dentro de ellas, diez pertenecen a la ERE cafésalario y siete UF a la ERE salario-café. Contrariamente de las UF sin casos de paludismo, sólo son 22 las que venden trabajo asalariado, con 2348 jornales contra 4520 de las UF con casos de paludismo.

En las mismas Tablas, observamos que, como indicadores de mayor capacidad económica, el número de UF, el número de jornales y el total de egresos invertidos en la compra de trabajo asalariado y, por tanto, con menores ries- 
Tabla 4

Unidades familiares con casos de paludismo, entre 1987 y 1993, con parcelas y/o actividades en los "trabajaderos" dentro de estrategias de reproducción económica. Yibeljoj, Chenalhó, Chiapas, 1994.

\begin{tabular}{|c|c|c|c|c|c|c|c|c|c|c|c|}
\hline \multirow[t]{3}{*}{ Estrategias } & \multirow{2}{*}{\multicolumn{2}{|c|}{ Con parcelas de maíz }} & \multirow{2}{*}{\multicolumn{2}{|c|}{ Con acahuales }} & \multicolumn{7}{|c|}{ Actividades en Maiz-Frijol } \\
\hline & & & & & \multicolumn{2}{|c|}{ ciclo $1^{*}$} & \multicolumn{2}{|c|}{ ciclo $2 * *$} & \multicolumn{3}{|c|}{ trabajo asalariado } \\
\hline & UF & $\%$ & UF & $\%$ & UF & $\%$ & UF & $\%$ & UF & Jornal & $\%$ \\
\hline café-maíz & 3 & 37,5 & 2 & 33,3 & 5 & 27,8 & 5 & 27,8 & 3 & 35 & 1,0 \\
\hline café-salario & 1 & 12,5 & 0 & 0 & 5 & 27,8 & 5 & 27,8 & 5 & 128 & 43,7 \\
\hline salario-café & 2 & 25,0 & 2 & 33,3 & 3 & 16,7 & 3 & 16,7 & 3 & 97 & 33,1 \\
\hline salario-maíz & 1 & 12,5 & 1 & 16,7 & 3 & 16,7 & 3 & 16,7 & 1 & 40 & 13,5 \\
\hline artesanía-maíz & 1 & 12,5 & 1 & 16,7 & 2 & 11,1 & 2 & 11,1 & 2 & 25 & 8,5 \\
\hline Totales & 8 & 100 & 6 & 100 & 18 & 100 & 18 & 100 & 14 & 3251 & 100 \\
\hline
\end{tabular}

Fuente: Encuesta socioeconómica del autor. $\mathrm{CIHMECH}, 1994$.

*Primavera-verano; **O toño-invierno; UF (Unidades Familiares)

Tabla 5

Unidades familiares sin casos de paludismo entre 1987 y 1993 con parcelas y/o actividades en los "trabajaderos", dentro de estrategias de reproducción económica. Yibeljoj, Chenalhó, Chiapas, 1994.

\begin{tabular}{|c|c|c|c|c|c|c|c|c|c|c|c|}
\hline \multirow[t]{3}{*}{ Estrategias } & \multirow{2}{*}{\multicolumn{2}{|c|}{ Con parcelas de maíz }} & \multicolumn{2}{|c|}{ Con acahuales } & \multicolumn{7}{|c|}{ Actividades en Maiz-Frijol } \\
\hline & & & \multirow[b]{2}{*}{ UF } & \multirow[b]{2}{*}{$\%$} & \multicolumn{2}{|c|}{ ciclo $1^{*}$} & \multicolumn{2}{|c|}{ ciclo $2^{* *}$} & \multicolumn{3}{|c|}{ trabajo asalariado } \\
\hline & UF & $\%$ & & & UF & $\%$ & UF & $\%$ & UF & Jorn & Ial \% \\
\hline artesanías-café & 1 & 50,0 & 4 & 66,6 & 4 & 40,0 & 4 & 66,6 & 0 & - & \\
\hline café-salario & 1 & 50,0 & 1 & 16,6 & 2 & 20,0 & 0 & 0 & 0 & - & \\
\hline salario-café & 0 & & 1 & 16,6 & 2 & 20,0 & 1 & 16,6 & 1 & 30 & 100 \\
\hline café-maíz & 0 & & 0 & 0 & 2 & 20,0 & 1 & 16,6 & 0 & - & \\
\hline café-frijol & 0 & & 0 & 0 & 0 & 0 & 0 & 0 & 0 & - & \\
\hline Totales & 2 & 100 & 6 & 100 & 10 & 100 & 6 & 100 & 1 & 30 & 100 \\
\hline
\end{tabular}

Fuente: Encuesta socioeconómica del autor. CIHMECH, 1994.

*Primavera-verano; **O toño-invierno; UF (Unidades Familiares)

Tabla 6

Estrategias de reproducción económica y compra-venta de fuerza de trabajo en unidades familiares con casos de paludismo, entre 1987 y 1993, en Yibeljoj, Chenalhó, Chiapas, 1994.

\begin{tabular}{lrrrrrrrrr}
\hline Estrategias & \multicolumn{8}{c}{$\begin{array}{c}\text { Venta } \\
\end{array}$} & \multicolumn{8}{c}{ Fuerza de Trabajo } \\
& UF J ornal & Ingreso* & $\%$ & UF & $\begin{array}{c}\text { Compra } \\
\text { J ornal }\end{array}$ & Egreso* & $\%$ \\
\hline café-maíz & 7 & 353 & 1765 & 7,8 & 10 & 736 & 3680 & 45,32 \\
café-salario & 10 & 1318 & 6590 & 29,16 & 9 & 410 & 2050 & 25,25 \\
salario-café & 7 & 1402 & 7010 & 31,02 & 3 & 96 & 480 & 5,91 \\
salario-maíz & 5 & 1342 & 6710 & 29,69 & 3 & 142 & 710 & 8,74 \\
artesanías-maíz & 3 & 105 & 525 & 2,33 & 4 & 240 & 1200 & 14,78 \\
Totales & 32 & 4520 & 22600 & 100,0 & 29 & 1624 & 8120 & 100,0 \\
\hline
\end{tabular}

UF (Unidades Familiares)

Fuente: Encuesta socioeconómica del autor. CIHMECH, 1994.

$* \mathrm{~N} \$$ (nuevos pesos) gos y prevalencia de paludismo, son mayores en las UF sin casos. Estos, como ya lo mencionamos, sólo son elementos indirectos de relación entre nivel económico de UF de casos y no casos - y dentro de ellos según estrategias de reproducción económica diferenciadas - y el paludismo. Si no, ver el dato interesante en la ERE sin casos de café-maíz, donde el segundo mayor porcentaje de compra de fuerza de trabajo se encuentra: 23,33\%; hablándonos esto de que, probablemente, tales UF tendrían parcelas de maíz en los “trabajaderos”, pero con poca o nula presencia de éstas UF en esos sitios de máximo riesgo de transmisión de la enfermedad palúdica. 
Estrategias de Reproducción Económica (ERE), volúmenes de producción agrícola y fuentes de ingreso en Unidades Familiares (UF) con casos y sin casos de paludismo

Teniendo el estudio sobre estrategias de reproducción de los campesinos indígenas de una comunidad representativa del municipio de Chenalhó una orientación económica, en los siguientes párrafos se expondrá una síntesis cuantitativa por actividades económicas. Cuantitativa, por expresar números y cifras de volúmenes de los principales productos agrícolas (café, maíz y frijol), así como de ingresos por la actividad artesanal y la compra y venta de fuerza de trabajo.

Al analizar las Tablas 8 y 9 , se observa que el nivel de ingresos en la suma total anual, para toda la producción (agrícola y artesanal), difiere grandemente entre las UF con casos y sin ca-
Tabla 7

Estrategias de reproducción económica y compra-venta de fuerza de trabajo en unidades familiares sin casos de paludismo, entre 1987 y 1993, en Yibeljoj, Chenalhó, Chiapas, 1994.

\begin{tabular}{|c|c|c|c|c|c|c|c|c|}
\hline \multirow[t]{3}{*}{ Estrategias } & \multicolumn{8}{|c|}{ Fuerza de Trabajo } \\
\hline & \multicolumn{4}{|c|}{ Venta } & \multicolumn{4}{|c|}{ Compra } \\
\hline & UF & J ornal & Ingreso* & $\%$ & UF & J ornal & Egreso* & $\%$ \\
\hline artesanías-café & 4 & 81 & 405 & 3,45 & 13 & 825 & 4125 & 38,8 \\
\hline café-salario & 11 & 855 & 4275 & 36,42 & 10 & 463 & 2315 & 21,78 \\
\hline café-maíz & - & - & - & - & 5 & 496 & 2480 & 23,33 \\
\hline salario-café & 5 & 1347 & 6735 & 57,36 & 2 & 56 & 280 & 2,64 \\
\hline café-frijol & 2 & 65 & 325 & 2,77 & 4 & 286 & 1430 & 13,45 \\
\hline Totales & 22 & 2348 & 117401 & 100,0 & 34 & 2126 & 106301 & 100,0 \\
\hline
\end{tabular}

Fuente: Encuesta socioeconómica del autor. CIHMECH, 1994.

* N \$ (nuevos pesos); UF (Unidades Familiares)

Tabla 8

Estrategias de reproducción económica, volumen e ingreso anual en unidades familiares con casos de paludismo, entre 1987 y 1993, en Yibeljoj, Chenalhó, Chiapas, 1994.

\begin{tabular}{lccccccccc}
\hline Estrategias & UF & $\begin{array}{c}\text { Café } \\
(\mathrm{Q} q)^{* *}\end{array}$ & $\begin{array}{c}\text { Ingreso } \\
\text { café* }\end{array}$ & $\begin{array}{c}\text { Maíz } \\
(\mathrm{Kg})\end{array}$ & $\begin{array}{c}\text { Ingreso } \\
\text { maíz* }\end{array}$ & $\begin{array}{c}\text { Frijol } \\
(\mathrm{Kg})\end{array}$ & $\begin{array}{c}\text { Ingreso } \\
\text { frijol* }\end{array}$ & $\begin{array}{c}\text { Ingreso* } \\
\text { artesanías }\end{array}$ & $\begin{array}{c}\text { Ingreso } \\
\text { total* }\end{array}$ \\
\hline café-maíz & 11 & 93 & 19094 & 6750 & 5737,5 & 485 & 1212,5 & 60 & 26104 \\
café-salario & 10 & 50 & 9663 & 4200 & 3570 & 180 & 450 & 180 & 13863 \\
salario-café & 7 & 16 & 3708 & 2675 & 2274 & 136 & 340 & 0 & 6322 \\
salario-maíz & 5 & 3 & 540 & 1575 & 1338,75 & 50 & 125 & 0 & 2003,75 \\
artesanías-maíz & 4 & 4 & 720 & 2050 & 1742,5 & 125 & 312,5 & 17000 & 19775 \\
Totales & 37 & 166 & 33725 & 17250 & 14662,75 & 976 & 2440 & 17240 & 68067,75 \\
\hline
\end{tabular}

Fuente: Encuesta socioeconómica del autor. $\mathrm{CIHMECH}, 1994 . * \mathrm{~N} \$$ (nuevos pesos); UF (Unidades Familiares)

$* *(\mathrm{Q} q)=\mathrm{Q}$ uintal, medida de peso equivalente a $60 \mathrm{Kg}$ de café en pergamino

Tabla 9

Estrategias de reproducción económica, volumen e ingreso anual en unidades familiares sin casos de paludismo, entre 1987 y 1993, en Yibeljoj, Chenalhó, Chiapas, 1994.

\begin{tabular}{lrrrrrrrrr}
\hline Estrategias & UF & $\begin{array}{c}\text { Café } \\
(\mathrm{Q} q)\end{array}$ & $\begin{array}{c}\text { Ingreso } \\
\text { café* }\end{array}$ & $\begin{array}{c}\text { Maíz } \\
(\mathrm{Kg})\end{array}$ & $\begin{array}{c}\text { Ingreso } \\
\text { maíz* }\end{array}$ & $\begin{array}{c}\text { Frijol } \\
(\mathrm{Kg})\end{array}$ & $\begin{array}{c}\text { Ingreso } \\
\text { frijol* }\end{array}$ & $\begin{array}{c}\text { Ingreso* } \\
\text { artesanías }\end{array}$ & $\begin{array}{c}\text { Ingreso } \\
\text { total* }\end{array}$ \\
\hline artesanías-café & 14 & 88 & 16264 & 680 & 578 & 550 & 1375 & 57300 & 75517 \\
café-salario & 11 & 68 & 12894 & 490 & 416,5 & 320 & 800 & 100 & 14210,5 \\
salario-café & 5 & 5 & 1380 & 125 & 312,5 & 180 & 153 & 150 & 1995,5 \\
café-maíz & 5 & 21 & 3942 & 275 & 233,75 & 0 & 0 & 0 & 4175,75 \\
café-frijol & 4 & 34 & 6210 & 360 & 306 & 300 & 750 & 0 & 7266 \\
Totales & 39 & 216 & 40690 & 1930 & 1836,75 & 1350 & 3078 & 57550 & 103154,75 \\
\hline
\end{tabular}

Fuente: Encuesta socioeconómica del autor. CIHMECH, 1994.

$* \mathrm{~N} \$$ (nuevos pesos); UF (Unidades Familiares) 
Estrategias de reproducción económica y porcentajes de ingreso en unidades familiares con paludismo, entre 1987 y 1993, en Yibeljoj, Chenalhó, Chiapas, 1994.

\begin{tabular}{|c|c|c|c|c|c|}
\hline \multirow{2}{*}{ Estrategias } & \multicolumn{2}{|c|}{ UF } & \multicolumn{3}{|c|}{$\%$ del ingreso } \\
\hline & $\mathrm{n}$ & $\%$ & UF & 1 & 2 \\
\hline café-maíz & 11 & 23,4 & 87,3 & 66,1 & 21,2 \\
\hline café-salario & 10 & 21,3 & 79,4 & 47,6 & 31,8 \\
\hline salario-café & 7 & 14,9 & 80,9 & 54,2 & 26,7 \\
\hline salario-maíz & 5 & 10,6 & 86,5 & 59,8 & 26,7 \\
\hline artesanías-maíz & 5 & 10,6 & 92,3 & 83,3 & 9,0 \\
\hline otras estrategias & 9 & 19,2 & 92,9 & 73,5 & 19,4 \\
\hline Totales & 47 & 100,0 & 86,5 & 84,1 & $\$ 2,5$ \\
\hline
\end{tabular}

Fuente: Encuesta socioeconómica del autor. CIHMECH, 1994. UF (Unidades Familiares)

Tabla 11

Estrategias de reproducción económica y porcentajes de ingreso en unidades familiares sin paludismo entre 1987 y 1993 en Yibeljoj, Chenalhó, Chiapas, 1994.

\begin{tabular}{|c|c|c|c|c|c|}
\hline \multirow{2}{*}{$\begin{array}{c}\text { Estrategias } \\
1\end{array}$} & \multicolumn{2}{|c|}{ UF } & \multicolumn{3}{|c|}{$\%$ del ingreso } \\
\hline & $n$ & $\%$ & UF & 1 & 2 \\
\hline artesanías-café & 14 & 29,8 & 97,6 & 76,3 & 21,3 \\
\hline café-salario & 11 & 23,4 & 95,1 & 71,5 & 23,9 \\
\hline salario-café & 5 & 10,6 & 95,4 & 74,8 & 19,0 \\
\hline café-maíz & 5 & 10,6 & 100,0 & 94,2 & 5,8 \\
\hline café-frijol & 4 & 8,5 & 93,7 & 78,4 & 15,3 \\
\hline otras estrategias & 8 & 17,0 & 92,4 & 75,4 & 17,0 \\
\hline Totales & 47 & 100,0 & 5,70 & $\not 88,4$ & $\$ 7,0$ \\
\hline
\end{tabular}

Fuente: Encuesta socioeconómica del autor. CIHMECH, 1994. UF (Unidades Familiares)

sos, esto independientemente del tipo de ERE respectivas: $\mathrm{N} \$ 68.067,70$ nuevos pesos al año para los casos, contra $\mathrm{N} \$ 103.164,70$ de los no casos. Si bien es cierto que en estas Tablas, no se incluyó el ingreso por venta de fuerza de trabajo, el que es favorable a las UF de casos: N\$ 22.600 contra N\$11.740 de las UF sin casos (Tablas 6 y 7). Dicha diferencia está lejos de compensar la diferencia aquella de ingreso por producción agrícola y artesanal, entre la sumatoria de ERE de casos y sin casos.

Al observar el ingreso generado producto por producto, vemos algo contundente que solo viene a terminar de confirmar resultados e hipótesis ya mencionados a lo largo de este subcapítulo: por un lado, que las UF que se dedican más al cultivo del maíz son las pertenecientes a las ERE de los casos de paludismo, donde volúmenes e ingresos superan en casi 10 veces a las UF de ERE con maíz de los no ca- sos pues la producción es de 17.250Kg ( N\$ $1.4662,50$ ) en UF de casos, contra $1.930 \mathrm{Kg}$ ( $\mathrm{N} \$$ $1.846,75)$ en UF sin casos. En el café sucede al revés, las UF con estrategias que incluyen al café donde no existen casos de paludismo superan en producción e ingresos, aunque no tan ampliamente como en el maíz, a las UF con ERE de casos: Ia producción es de 216 Qq (N\$ 40.690) para UF sin casos contra 166 Qq (N\$ 33.725) de los UF con casos.

Finalmente, el frijol tiene resultados similares a los del maíz en volúmenes o ingresos favorables a las UF de casos, aunque no es tan amplia la diferencia. La artesanía, como se puede observar en las mismas Tablas, tiene ingresos muy superiores en las UF de no casos con esta estrategia artesanía-café, en relación a las UF con casos de la estrategia artesanía-maíz: $N \$ 57.550$ contra N\$17.240, respectivamente; por lo que se deduce que quienes se dedican a 
otra actividad no se enferman de paludismo, dado que el riesgo se reduce.

Una reflexión analítica general es conveniente, al observarse los resultados de estas últimas Tablas (Tablas 6,7,8 y 9), sobre todo al revisar las estrategias de reproducción económica incluidas en los casos y no casos de paludismo. Las UF de casos de paludismo que se desenvuelven en las ERE de café-maíz, café-salario y salario-café serían, por el número de UF y por el carácter de sus principales actividades económicas - el cultivo y producción de maíz y el trabajo como peones asalariados -, las más expuestas a las condiciones agroecológicas de transmisión del paludismo. Contrariamente, de las UF sin casos que tendrían menos riesgos de infección de paludismo serían, por número de UF y tipo de actividad económica, las que se desenvuelven en las estrategias artesanía-café y café-salario. Esta última estrategia, a pesar de tener actividad de trabajo asalariado, este se realiza fuera de los "trabajaderos" de maíz-frijol (Tabla 5).

Al analizar específicamente la contribución porcentual de cada componente del total de estrategias, tanto de las UF con casos como las de no casos, observamos en las Tablas $10 \mathrm{y}$ 11 lo siguiente: Ios porcentajes de los totales de ingreso de las UF de las ERE de casos y no casos de paludismo, cuando sumados a los porcentajes de los dos componentes del ingreso de cada una de las estrategias, resultan de relativa alta homogeneidad (sobre todo los de no casos), entre $79,4 \%$ de los de café-trabajo asalariado y $92,9 \%$ de los de otras estrategias en los de casos $y$, entre $92,4 \%$ de las de otras estrategias y $100 \%$ en la de café-maíz de los no casos. Esto lo consignamos sólo para dar cuenta de que, para todas las ERE (con casos y sin casos), los dos componentes económicos de todas y cada una de las estrategias tienen una al ta y semejante importancia como fuente de ingreso.

La relación del paludismo con los valores porcentuales consignados como porcentajes sumados de los dos componentes, en las dos ERE mas importantes en casos y no casos, así como vistos separadamente, en todo caso, sólo nos confirmaría lo dicho en la reflexión analítica acerca de las Tablas 6,7,8 y 9.

\section{Referencias}

FERNANDEZ DE CASTRO, P., 1988. Panorama Histórico Epidemiológico del Paludismo en México. México: Secretaría de Salud.

FORATTINI, O. P., 1962. Entomologia Médica. Vol.1, São Paulo: Faculdade de Higiene e Saúde Pública.

GONZALEZ-CASANOVA, H. P., 1994. Un gran fiasco en el control del paludismo: El caso de Chiapas, México. Revista ClHMECH, 3:35-65.

LOYOLA, E. G., 1985. El paludismo: ¿Un problema de Salud Pública? Econoticias: 3a Época, 9:1-3.

MERA, O. L. M., 1989. Condiciones naturales para la producción. In: El Subdesarrollo Agrícola en Los Altos deChiapas ( V. R., Parra, Coordinador). México: UACH.

OPS-OMS (Organización Panamericana de la Salud Organización Mundial de Salud), 1990. Las condiciones de salud en las Américas: Enfermedades Transmisibles. Washington, DC: OPS-OMS

SSA (Secretaria de Salud), 1993. Programa deVigilancia Epidemiológica. San Cristóbal de Las Casas: Jurisdicción Sanitária II. 